\title{
Some Classes of Generating Functions for the Laguerre and Hermite Polynomials
}

\author{
By M. E. Cohen
}

\begin{abstract}
In the first half of the article, we present two theorems which give, as special cases, a number of new classes of generating functions for the Laguerre polynomial. These formulae extend the recent results of Carlitz [2] and others. The latter part of our work deals with two theorems involving new generating functions for the Hermite and generalized Hermite polynomials, thus generalizing some well-known expansions. The method of proof adopted in this paper differs from that of previous workers.
\end{abstract}

I. Introduction. Extending the work done by Chatterjea [3] and Brown [1], Carlitz [2] gave the generating function

$$
\sum_{n=0}^{\infty} t^{n} L_{n}^{a+b n}(x)=\frac{(1+v)^{a+1}}{1-b v} \exp (-x v),
$$

where $v$ satisfies $v=t(1+v)^{b+1}, v(0)=0$, and $a, b$ are arbitrary complex numbers. The Laguerre polynomial is defined in the usual way [6, Volume II, p. 188] .

In our presentation, we derive two theorems which give expansions for the Laguerre polynomial. A special case of Theorem 1 is seen to be

$$
\begin{aligned}
& \sum_{n=0}^{\infty} t^{n} L_{n}^{v+b n}[x(1+a n)] \\
& \quad=(1-z)^{1-v}\left[1-z(b+2-a x)+z^{2}(b+1)\right]^{-1} \exp [x z /(z-1)],
\end{aligned}
$$

where $t=z(1-z)^{b} \exp [a x z /(1-z)], v, a, b$ are arbitrary complex numbers and $|t|<$ 1. Letting $a=0$ in (1.2) gives essentially (1.1).

Theorem 2 yields a number of new expressions. One class of generating functions is

$$
\sum_{n=0}^{\infty} \frac{t^{n}}{(v+b n+n)} L_{n}^{v+b n}[x(1+a n)]
$$

$$
=(v)^{-1}(1-z)^{-v} \exp [x z /(z-1)]_{1} F_{1}\left[\begin{array}{cc}
1 ; & \frac{x z(1+b-a v)}{(1-z)(1+b)} \\
\frac{v+1+b}{1+b} ; &
\end{array}\right]
$$

where $t=z(1-z)^{b} \exp [a x z /(1-z)]$ and $|t|<1$.

Received April 29, 1976; revised September 13, 1976.

AMS (MOS) subject classifications (1970). Primary 42A56; Secondary 33A65.

$\mathrm{Key}$ words and phrases. Generating functions, Hermite polynomial, hypergeometric function, incomplete gamma function, Laguerre polynomial, operators. 
The right-hand side of (1.3) is the incomplete gamma function. See Chapter IV of Luke [9] for extensive treatment and bibliography of this function. A reduction of interest involves the case of $1+b=a v$, and we obtain

$$
\sum_{n=0}^{\infty} \frac{t^{n}}{(1+a n)} L_{n}^{v+a v n-n}[x(1+a n)]=(1-z)^{-v} \exp [x z /(z-1)],
$$

where $t=z(1-z)^{a v-1} \exp [a x z /(1-z)]$ and $|t|<1$. For $a=0$ in (1.3), we deduce the known result and its extension given by Eq. (4.13) of [4] .

A second class of generating functions, a consequence of Theorem 2, may be expressed as

$$
\begin{aligned}
\sum_{n=0}^{\infty} \frac{t^{n}}{(1+a n)} L_{n}^{v+b n}[x(1+a n)] & {\left[\begin{array}{cc}
\frac{1+b-a v}{a}, & 1 ; \\
& =(1-z)^{-v} \exp [x z /(z-1)]_{2} F_{1}\left[\begin{array}{cc}
a+1 \\
a
\end{array}\right.
\end{array}\right] }
\end{aligned}
$$

where $t=z(1-z)^{b} \exp [a x z /(1-z)]$ and $|t|<1$. The right-hand side of (1.5) is the incomplete beta function [9, p. 299]. Putting $b=a+a v$, we have a simplification of interest:

$$
\sum_{n=0}^{\infty} \frac{t^{n}}{(1+a n)} L_{n}^{v+a n+a v n}[x(1+a n)]=(1-z)^{-1-v} \exp [x z /(z-1)]
$$

where $t=z(1-z)^{a+a v} \exp [a x z /(1-z)]$ and $|t|<1$. Letting $a \rightarrow 0$ in (1.5) gives the known Carlitz expansion (1.1).

A third class of generating functions may be deduced from Theorem 2, which assumes the form

$$
\begin{aligned}
& \sum_{n=0}^{\infty} \frac{t^{n}}{(n+1)} L_{n}^{v+b n}[x(1+a n)] \\
&=\frac{\exp [(-x z+x-a x) /(1-z)]}{(1-z)^{v} z(v-b)}\left\{{ }_{1} F_{1}\left[\begin{array}{cc}
v-b ; & x(a-1) \\
v-b+1 ; & 1-z
\end{array}\right]\right. \\
&\left.-(1-z)^{v-b}{ }_{1} F_{1}\left[\begin{array}{cc}
v-b ; & x(a-1) \\
v-b+1 ; &
\end{array}\right]\right\}
\end{aligned}
$$

where $t=z(1-z)^{b} \exp [a x z /(1-z)]$ and $|t|<1$.

The three classes of generating functions given above are in fact particular examples of the more general expansion 


$$
\begin{aligned}
\sum_{n=0}^{\infty} & \frac{t^{n} L_{n}^{v+b n}[x(1+a n)]}{(l-v-b n-n)((l-v+s-b n-n) /(s))_{l^{\prime}}} \\
(1.8)= & \frac{(1-z)^{l-v} \exp [x z /(z-1)]}{l^{\prime} !} \sum_{r=0}^{l^{\prime}} \sum_{n=0}^{l+s r} \frac{(-z)^{n}(1-z)^{r s-n}\left(-l^{\prime}\right)_{r}(-l-r s)_{n}}{n ! r !(l-v+r s-b n-n)} \\
& \cdot{ }_{1} F_{1}\left[\begin{array}{cc}
(v-l+b+1+b n+n-r s) /(b+1) ; & \left.\frac{x z(b+1+a l-a v+a r s)}{(1-z)(b+1)}\right],
\end{array}\right.
\end{aligned}
$$

where $t=z(1-z)^{b} \exp [a x z /(1-z)], v, a, b$ are arbitrary complex numbers, $l, l^{\prime}, s$ nonnegative integers, and $|t|<1$. Note that we have assumed $l, l^{\prime}$, and $s$ to be nonnegative integers to terminate the series on the right-hand side of (1.8). This condition is not necessary. The result is valid provided both sides of (1.8) exist for arbitrary values of the parameters involved.

Letting $a=0$ and $l^{\prime}=0$ in (1.8) gives essentially Eq. (3.4) of Zeitlin [10] .

As the Bessel polynomial is expressed as [6, Volume I, p. 195] and the Poisson-Charlier polynomial as [6, Volume I, p. 268], then the formulae given for the Laguerre polynomial may be converted to generate the above-mentioned polynomials.

Theorems 3 and 4 involve the Hermite and generalized Hermite polynomials. A special case is the generating function

$$
\begin{aligned}
& \sum_{n=0}^{\infty} \frac{(1+b n)^{1 / 2 n}}{n !} t^{n} H_{n}\left[x(1+a n) /(1+b n)^{1 / 2}\right] \\
& \quad=\exp \left[-z^{2}-2 x z\right]\left[1+2 b z^{2}+2 a x z\right]^{-1}
\end{aligned}
$$

where $t=(-z) \exp \left[b z^{2}+2 a x z\right]$ and $\left|2 a x z \exp \left[b z^{2}+2 a x z+1\right]\right|<1$. The Hermite polynomial is defined in [6, Volume II, p. 193]. The special case $a=b=$ 0 in (1.9) gives the old and well-known result [6, Volume II, p. 194, Eq. (19)]. If $b=0$ and $a=0$ in (1.9), then the equations (2.14) and (2.16), respectively, of Cohen [4], present themselves.

From Theorem 4, one class of new generating functions that may be deduced is

$$
\begin{aligned}
& \sum_{n=0}^{\infty} \frac{t^{n}(1+b n)^{1 / 2(n-2)}}{n !} H_{n}\left[x(1+a n) /(1+b n)^{1 / 2}\right] \\
& \quad=\exp \left[-z^{2}-2 x z\right]_{1} F_{1}\left[\begin{array}{cc}
1 ; & 2 x z(b-a) / b \\
\frac{b+1}{b} ; &
\end{array}\right],
\end{aligned}
$$

where $t=(-z) \exp \left(b z^{2}+2 a x z\right)$ and $\left|2 a x z \exp \left(b z^{2}+2 a x z+1\right)\right|<1$. If $b \rightarrow 0$ and $a \rightarrow 0$ in (1.10), then Eqs. (2.14) and (2.17), respectively, of [4] are obtained. A special case of interest may be derived from (1.10) for $a=b$ to give 


$$
\sum_{n=0}^{\infty} \frac{t^{n}(1+a n)^{1 / 2(n-2)}}{n !} H_{n}\left[x(1+a n)^{1 / 2}\right]=\exp \left[-z^{2}-2 x z\right],
$$

where $t=(-z) \exp \left(a z^{2}+2 a x z\right)$ and $\left|2 a x z \exp \left[a z^{2}+2 a x z+1\right]\right|<1$.

A second class of generating functions is a special case of Theorem 4, and may be expressed as

$$
\sum_{n=0}^{\infty} \frac{t^{n}(1+b n)^{1 / 2 n}}{n !(1+a n)} H_{n}\left[x(1+a n) /(1+b n)^{1 / 2}\right]
$$

$$
=\exp \left[-z^{2}-2 x z\right]_{1} F_{1}\left[\begin{array}{c}
1 ; \\
\frac{1+2 a}{2 a} ;
\end{array} \quad z^{2}(a-b) / a\right],
$$

where $t=(-z) \exp \left[b z^{2}+2 a x z\right]$ and $\left|2 a x z \exp \left[b z^{2}+2 a x z+1\right]\right|<1$. Letting $b \rightarrow$ 0 and $a \rightarrow 0$ in (1.12) give the known Eqs. (2.15) and (2.16), respectively, of [4] .

2. Generating Functions. The method of proof in the four theorems is a modification and extension of the one employed in obtaining new generating functions for the Jacobi polynomial in [5]. As in [5], no appeal is made to the Lagrange Theorem. It differs from that of previous workers, including the approach in [4].

THEOREM 1. For $\alpha, \beta$, s arbitrary complex numbers and $r$ a positive integer

$$
\begin{gathered}
\sum_{k=0}^{\infty} \frac{t^{k}(\alpha+1+s k)_{k}}{k !}{ } F_{r}\left[\begin{array}{cc}
\Delta(-k, r) ; & x(\beta+s k) \\
\Delta(-\alpha-s k-k, r) ;
\end{array}\right] \\
=(1-z)^{\alpha+1}(1+s z+r s y)^{-1} \exp [-\beta y]
\end{gathered}
$$

where $t=(-z)(1-z)^{-s-1} \exp (s y), x=(-y)[(z-1) / z]^{r},(a)_{k}=\Gamma(a+k) / \Gamma(a)$, quotient of gamma functions $=a(a+1)(a+2) \ldots(a+k-1)$ for $k$ a positive integer, and $\Delta(-k, r)=-k / r,(-k+1) / r, \ldots,(-k+r-1) / r .|t|<1$, and ${ }_{r} F_{r}$ is the generalized hypergeometric polynomial $[9, p .155]$.

Proof. Let us begin with the expression

$$
\sum_{m=0}^{\infty} \sum_{n=0}^{\infty} \frac{z^{n} y^{m}}{n ! m !} D^{n}\left[x^{\alpha-\beta+n} \delta^{m}\left\{x^{\beta}\left(1-x^{s}\right)^{n}\left(1-x^{s^{\prime}}\right)^{m}\right\}\right],
$$

where $D \equiv d / d x$ and $\delta \equiv x d / d x$. Now

$$
\begin{aligned}
D^{n} & {\left[x^{\alpha-\beta+n} \delta^{m}\left\{x^{\beta}\left(1-x^{s}\right)^{n}\left(1-x^{s^{\prime}}\right)^{m}\right\}\right] } \\
& =\sum_{k=0}^{n} \sum_{p=0}^{m} \frac{(-n)_{k}(-m)_{p}\left(\beta+s k+s^{\prime} p\right)^{m}\left(\alpha+1+s k+s^{\prime} p\right)_{n} x^{\alpha+s k+s^{\prime} p}}{k ! p !}
\end{aligned}
$$

Taking (2.3) and (2.4) for $x=1$, and putting the results in (2.2), one has 


$$
\begin{aligned}
\sum_{m=0}^{\infty} \sum_{n=0}^{\infty} \sum_{p=0}^{m} \sum_{k=0}^{n} \frac{z^{n} y^{m}(-n)_{k}(-m)_{p}\left(\beta+s k+s^{\prime} p\right)^{m}\left(\alpha+1+s k+s^{\prime} p\right)_{n}}{k ! p ! n ! m !} \\
\quad=\sum_{m=0}^{\infty} \sum_{n=0}^{\infty} \frac{(-s z)^{n}\left(-s^{\prime} y\right)^{m}(m+n) !}{n ! m !} \\
=\left[1+s z+s^{\prime} y\right]^{-1} .
\end{aligned}
$$

We go from (2.6) to (2.7) using the binomial expansions. Now applying the series transformation

$$
\sum_{m=0}^{\infty} \sum_{n=0}^{\infty} \sum_{p=0}^{m} \sum_{k=0}^{n} f(n, m, k, p)=\sum_{n, m, k, p=0}^{\infty} f(n+k, m+p, k, p)
$$

to (2.5), and simplifying, gives

$$
\sum_{k=0}^{\infty} \sum_{p=0}^{\infty} \frac{(-z)^{k}(-y)^{p} \exp \left[y\left(\beta+s k+s^{\prime} p\right)\right]\left(\beta+s k+s^{\prime} p\right)^{p}\left(\alpha+1+s k+s^{\prime} p\right)_{k}}{k ! p !(1-z)^{\alpha+1+s k+k+s^{\prime} p}}
$$

The summation of the series over $n$ and $m$ is achieved with the aid of the binomial and exponential expansions. Equating (2.7) and (2.9), and employing

$$
\sum_{p=0}^{\infty} \sum_{k=0}^{\infty} f(p, k)=\sum_{k=0}^{\infty} \sum_{p=0}^{[r / k]} f(p, k-r p)
$$

with $r=s^{\prime} / s$, and some algebra, gives the required theorem.

THEOREM 2. For $\alpha, \beta, s, s^{\prime}$ arbitrary complex numbers, $l, l^{\prime}, s^{\prime \prime}$ nonnegative integers and $r$ a positive integer

$$
\begin{gathered}
\sum_{k=0}^{\infty} \frac{(\alpha+1+s k)_{k} t^{k}}{(\alpha+l+1+s k) k !\left(\left(\alpha+l+1+s^{\prime \prime}+s k\right) / s^{\prime \prime}\right)_{l^{\prime}}} \\
\cdot{ }_{r} F_{r}\left[\begin{array}{cc}
\Delta(-k, r) ; & x(\beta+s k) \\
\Delta(-\alpha-s k-k, r) ;
\end{array}\right.
\end{gathered}
$$

$$
\begin{aligned}
& =\frac{(1-z)^{\alpha+l+1} \exp (-\beta y)}{l^{\prime} !} \cdot \sum_{q=0}^{l^{\prime}} \sum_{k=0}^{l+s^{\prime \prime} q} \frac{(-z)^{k}(1-z)^{s^{\prime \prime} q-k}\left(-l^{\prime}\right)_{q}\left(-l-s^{\prime \prime} q\right)_{k}}{k ! q !\left(\alpha+l+1+s^{\prime \prime} q+s k\right)} \\
& \cdot{ }_{1} F_{1}\left[\begin{array}{cc}
\left(\alpha+l+1+s^{\prime}+s^{\prime \prime} r+s k\right) / s^{\prime} ; & \left.y\left(\beta-\alpha-l-1-s^{\prime \prime} q\right)\right],
\end{array}\right.
\end{aligned}
$$

where $t=(-z)(1-z)^{-s-1} \exp (s y), x=(-y)[(z-1) / z]^{r}$, and $|t|<1$. Other symbols are defined in Theorem 1.

Proof. We first evaluate the integral 


$$
\int_{0}^{1} x^{l}\left(1-x^{s^{\prime \prime}}\right)^{l^{\prime}} D^{n}\left[x^{\alpha-\beta+n} \delta^{m}\left\{x^{\beta}\left(1-x^{s}\right)^{n}\left(1-x^{s^{\prime}}\right)^{m}\right\}\right] d x
$$

Expanding the operators as in Theorem 1, we have

$$
\begin{gathered}
\sum_{p=0}^{m} \sum_{k=0}^{n} \frac{(-n)_{k}(-m)_{p}\left(\beta+s k+s^{\prime} p\right)^{m}\left(\alpha+1+s k+s^{\prime} p\right)_{n}}{k ! p !} \\
\cdot \int_{0}^{1} x^{s k+s^{\prime} p+l}\left(1-x^{s^{\prime \prime}}\right)^{\prime \prime} d x .
\end{gathered}
$$

The integral in (2.13) may be evaluated to give

$$
\frac{l^{\prime} !}{\left(\alpha+l+1+s k+s^{\prime} p\right)\left(\left(\alpha+l+1+s^{\prime \prime}+s k+s^{\prime} p\right) / s^{\prime \prime}\right) l^{\prime}} \text {. }
$$

Going back to $(2.12)$, and expanding $\left(1-x^{s^{\prime \prime}}\right)^{l^{\prime}}$ gives

$$
\sum_{q=0}^{l^{\prime}} \frac{\left(-l^{\prime}\right)_{q}}{q !} \int_{0}^{1} x^{l+s^{\prime \prime} q} D^{n}\left[x^{\alpha-\beta+n} \delta^{m}\left\{x^{\beta}\left(1-x^{s}\right)^{n}\left(1-x^{s^{\prime}}\right)^{m}\right\}\right] d x .
$$

Now integrating by parts $n$ times, then expanding $\left(1-x^{s}\right)^{n}$ and $\left(1-x^{s^{\prime}}\right)^{m}$ and integrating again, $(2.15)$ reduces to

$$
\sum_{q=0}^{l^{\prime}} \sum_{p=0}^{m} \sum_{k=0}^{n} \frac{\left(-l^{\prime}\right)_{q}\left(-l-s^{\prime \prime} q\right)_{n}(-n)_{k}(-m)_{p}\left(\beta+s k+s^{\prime} p\right)^{m}}{q ! p ! k !\left(\alpha+l+1+s^{\prime \prime} q+s k+s^{\prime} p\right)} .
$$

Equating (2.13) and (2.14) to (2.16), multiplying both sides of the resulting equation by

$$
\sum_{m=0}^{\infty} \sum_{n=0}^{\infty} \frac{z^{n} y^{m}}{n ! m !}
$$

and proceeding as in Theorem 1, we have, after simplification

$$
\begin{gathered}
\sum_{p=0}^{\infty} \sum_{k=0}^{\infty} \frac{(-z)^{k}(-y)^{p} \exp \left[y\left(\beta+s k+s^{\prime} p\right)\right]\left(\beta+s k+s^{\prime} p\right)^{p}\left(\alpha+1+s k+s^{\prime} p\right)_{k}}{k ! p !(1-z)^{\alpha+1+s k+k+s^{\prime} p}\left(\alpha+1+l+s k+s^{\prime} p\right)\left(\left(\alpha+l+s^{\prime \prime}+1+s k+s^{\prime} p\right) / s^{\prime \prime}\right)_{l^{\prime}}} \\
=\frac{1}{l^{\prime} !} \sum_{q=0}^{l^{\prime}} \sum_{k=0}^{l+s^{\prime \prime} q} \frac{(-z)^{k}(1-z)^{l+s^{\prime \prime} q-k}\left(-l^{\prime}\right)_{q}\left(-l-s^{\prime \prime} q\right)_{k}}{k ! q !\left(\alpha+l+1+s^{\prime \prime} q+s k\right)} \\
\cdot{ }_{1} F_{1}\left[\begin{array}{c}
1 ; \\
\left(\alpha+l+s^{\prime}+1+s^{\prime \prime} q+s k\right) / s^{\prime} ;
\end{array} \quad y\left(\beta-\alpha-l-1-s^{\prime \prime} q\right)\right] .
\end{gathered}
$$

Applying the series transformation (2.10) and simplifying, gives Theorem 2 .

THEOREM 3. For $\alpha \beta, s^{\prime}$ arbitrary complex numbers and $r$ a positive integer

$$
\begin{aligned}
\sum_{p=0}^{\infty} \frac{t^{p}\left(\beta+s^{\prime} p\right)^{p}}{p !} F_{0} & {\left[\Delta(-p, r) ;-; \frac{x r^{r}\left(\alpha+s^{\prime} p\right)}{\left(\beta+s^{\prime} p\right)^{r}}\right] } \\
& =\exp (-\beta y-\alpha z)\left(1+s^{\prime} y+r s^{\prime} z\right)^{-1}
\end{aligned}
$$


where $t=(-y) \exp \left(s^{\prime} y+s^{\prime} z\right), x=(-z)(y)^{-r}$, and $\left|s^{\prime} y \exp \left(s^{\prime} y+s^{\prime} z+1\right)\right|<1$. See Theorem 1 for other symbols.

Proof. Consider

$$
\sum_{m=0}^{\infty} \sum_{n=0}^{n} \frac{z^{n} y^{m}}{n ! m !} \delta^{n}\left[x^{\alpha-\beta} \delta^{m}\left\{x^{\beta}\left(1-x^{s}\right)^{n}\left(1-x^{s^{\prime}}\right)^{m}\right\}\right]
$$

At $x=1$ we have, after expansion

$$
\sum_{m=0}^{\infty} \sum_{n=0}^{\infty} \frac{z^{n} y^{m}}{n ! m !} \sum_{p=0}^{m} \sum_{k=0}^{n} \frac{(-n)_{k}(-m)_{p}\left(\alpha+s k+s^{\prime} p\right)^{n}\left(\beta+s k+s^{\prime} p\right)^{m}}{k ! p !}
$$

But at $x=1,(2.19)$ also reduces to

$$
\sum_{m=0}^{\infty} \sum_{n=0}^{\infty} \frac{(-s z)^{n}\left(-s^{\prime} y\right)^{m}(n+m) !}{n ! m !} .
$$

Equating (2.20) to (2.21), and proceeding as in Theorem 1, with applying series transformations and simplifying, gives

$$
\begin{gathered}
\sum_{p=0}^{\infty} \sum_{k=0}^{\infty} \frac{(-z)^{k}(-y)^{p} \exp \left[y\left(\beta+s k+s^{\prime} p\right)+z\left(\alpha+s k+s^{\prime} p\right)\right]\left(\alpha+s k+s^{\prime} p\right)^{k}\left(\beta+s k+s^{\prime} p\right)^{p}}{k ! p !} \\
=\left(1+z s+y s^{\prime}\right)^{-1}
\end{gathered}
$$

Using the series transformation (2.10) and some manipulation results in Theorem 3. Note that for $s^{\prime}=0$ in the above theorem, the ${ }_{r} F_{0}$ hypergeometric polynomial is essentially the generalized Hermite polynomial which occurs in probability problems. It has been studied by Gould and Hopper [7], Gupta and Jain [8], Cohen [4], and others.

THEOREM 4. For $\alpha, \beta, s, s^{\prime}$ arbitrary complex numbers and $r, r^{\prime}$ positive integers (a)

$$
\sum_{p=0}^{\infty} \frac{\left(\beta+s^{\prime} p\right)^{p} t^{p}}{p !\left(\alpha+s^{\prime} p\right)} r F_{0}\left[\Delta(-p, r) ;-; \frac{x r^{r}\left(\alpha+s^{\prime} p\right)}{\left(\beta+s^{\prime} p\right)^{r}}\right]
$$

$$
=\frac{\exp (-\alpha z-\beta y)}{(\alpha)}{ }_{1} F_{1}\left[\begin{array}{c}
1 ; \\
\left(\alpha+s^{\prime}\right) / s^{\prime} ;
\end{array} y(\beta-\alpha)\right]
$$

where $t=(-y) \exp \left(s^{\prime} y+s^{\prime} z\right), x=(-z)(y)^{-r}$, and $\left|s^{\prime} y \exp \left(s^{\prime} y+s^{\prime} z+1\right)\right|<1$.

(b)

$$
\sum_{k=0}^{\infty} \frac{(\alpha+s k)^{k-1} t^{\prime k}}{k !}{ }_{r} F_{0}\left[\Delta\left(-k, r^{\prime}\right) ;-; \frac{r^{\prime r^{\prime}} x^{\prime}(\beta+s k)}{(\alpha+s k)^{r^{\prime}}}\right]
$$

$$
=\frac{\exp (-\alpha z-\beta y)}{(\alpha)}{ }_{1} F_{1}\left[\begin{array}{cc}
1 ; & y(\beta-\alpha) \\
\left(\alpha+r^{\prime} s\right) / r^{\prime} s ; &
\end{array}\right]
$$


where $t^{\prime}=(-z) \exp (s y+s z), x^{\prime}=(-y)(z)^{-r^{\prime}}$, and $|s z \exp (s y+s z+1)|<1$. Other symbols are defined in Theorem 1.

Proof. Let us evaluate the expression

$$
\sum_{m=0}^{\infty} \sum_{n=0}^{\infty} \frac{z^{n} y^{m}}{n ! m !} \int_{0}^{1} x^{-1} \delta^{n}\left[x^{\alpha-\beta} \delta^{m}\left\{x^{\beta}\left(1-x^{s}\right)^{n}\left(1-x^{s^{\prime}}\right)^{m}\right\}\right] d x .
$$

Expanding and operating as in the previous theorems, we have

$$
\sum_{m=0}^{\infty} \sum_{n=0}^{\infty} \frac{z^{n} y^{m}}{n ! m !} \sum_{p=0}^{m} \sum_{k=0}^{n} \frac{(-n)_{k}(-m)_{p}\left(\beta+s k+s^{\prime} p\right)^{m}\left(\alpha+s k+s^{\prime} p\right)^{n-1}}{k ! p !}
$$

Going back to (2.25) and integrating, we find the only contribution comes from $n=$ 0 and hence (2.25) may be evaluated to give

$$
\sum_{m=0}^{\infty} \frac{[y(\beta-\alpha)]^{m} m !}{m !(\alpha)\left(\left(\alpha+s^{\prime}\right) / s^{\prime}\right)_{m}}
$$

Equating (2.26) and (2.27), using the series transformation, and reducing the summation over $n$ and $m$, one obtains the expression

$$
\sum_{p=0}^{\infty} \sum_{k=0}^{\infty} \frac{(-z)^{k}(-y)^{p}\left(\beta+s k+s^{\prime} p\right)^{p}\left(\alpha+s k+s^{\prime} p\right)^{k-1} \exp \left[z\left(\alpha+s k+s^{\prime} p\right)+y\left(\beta+s k+s^{\prime} p\right)\right]}{k ! p !}
$$

$$
=(1 / \alpha)_{1} F_{1}\left[\begin{array}{cc}
1 ; \\
\left(\alpha+s^{\prime}\right) / s^{\prime} ;
\end{array}, y(\beta-\alpha)\right] .
$$

Now applying (2.10) to (2.28) twice, with $p$ and $k$ interchanged, and some algebra, gives the two parts of the theorem. Note that $r=s / s^{\prime}$, and $r^{\prime}=s^{\prime} / s$.

Department of Mathematics

California State University, Fresno

Fresno, California 93740

1. J. W. BROWN, “On zero type sets of Laguerre polynomials," Duke Math. J., v. 35, 1968, pp. 821-823. MR 38 \#2348.

2. L. CARLITZ, "Some generating functions for Laguerre polynomials," Duke Math. J., v. 35, 1968, pp. 825-827. MR 39 \#1700.

3. S. K. CHATTERJEA, "Some generating functions," Duke Math. J., v. 32, 1965, pp. 563564. MR 31 \#5989.

4. M. E. COHEN, "On expansion problems: New classes of formulas for the classical functions," SIAM J. Math. Anal, v. 7, 1976, pp. 702-712.

5. M. E. COHEN, "Generating functions for the Jacobi polynomial," Proc. Amer. Math. Soc., v. 57, 1976, pp. 271-275.

6. A. ERDELYI et al., Higher Transcendental Functions, Vols. I, II, McGraw-Hill, New York, 1953. MR 15, 419.

7. H. W. GOULD \& A. T. HOPPER, "Operational formulas connected with two generalizations of Hermite polynomials," Duke Math. J., v. 29, 1962, pp. 51-63. MR 24 \#A2689.

8. R. P. GUPTA \& G. C. JAIN, "A generalized Hermite distribution and its properties," SIAM J. Appl. Math., v. 27, 1974, pp. 359-363. MR 50 \#2580.

9. Y. L. LUKE, Mathematical Functions and Their Approximations, Academic Press, New York, 1975.

10. D. ZEITLIN, "A new class of generating functions for hypergeometric polynomials," Proc. Amer. Math. Soc., v. 25, 1970, pp. 405-412. MR 41\#8719. 